\title{
Melanoma drug wins US approval
}

Therapy is the first to extend lifespan in advanced cases.

\section{BY HEIDI LEDFORD}

A drug that takes an unusual approach to harnessing patients' immune systems to fight cancer has been approved by the US Food and Drug Administration (FDA). On 25 March, the FDA cleared ipilimumab, produced by Bristol-Myers Squibb, based in New York, to treat advanced melanoma, a particularly lethal form of skin cancer. Although the drug typically lengthens a patient's life by only 4 months or so, in clinical trials a fraction of patients lived much longer.

Ipilimumab marks success on two fronts: advanced melanoma, which has beaten multiple drug candidates, and cancer immunotherapy, which has had several high-profile disappointments in late-stage clinical trials.

Unlike other cancer immunotherapies on the market, which stimulate the immune system, ipilimumab lifts one of the immune system's restraints. The drug is an antibody that targets the protein cytotoxic T-lymphocyte antigen 4

(CTLA-4). CTLA-4 guards against autoimmune reactions by inhibiting some $T$ cells, but can also keep the immune system from attacking cancers. "The other therapies slam on the immune system's gas pedal," says Patrick Hwu, an oncologist at the MD Anderson Cancer Center in Houston, Texas. "Ipilimumab releases the brakes."

Hwu helped to conduct some of the first clinical trials of the drug more than five years ago. Although patients with advanced melanoma rarely survive more than a year past their diagnosis, some of those initial participants are still alive, he says, years after receiving their final dose of ipilimumab. This stands in stark contrast to the widely hailed genetically targeted melanoma drugs, called BRAF inhibitors, that are currently wending their way through clinical

D NATURE.COM For more see the Outlook on cancer prevention: go.nature.com/9fqrbn trials. The inhibitors produce dramatic responses in patients whose melanoma has mutations in the BRAF protein, but the cancer becomes resistant about seven months after starting the drug (see Nature 467, 140-141; 2010).

Although ipilimumab can add years of life, only $20-30 \%$ of patients show any benefit at all (F. S. Hodi et al. N. Engl. J. Med. 363, 711-723; 2010). "We clearly have to do much better than that," says Hwu. Furthermore, the drug caused severe or fatal side effects in almost 13\% of patients, prompting the FDA to qualify its approval with a mitigation strategy.

Ultimately, the key may be to combine ipilimumab with targeted therapies such as the BRAF inhibitors, says James Allison, an immunologist at the Memorial SloanKettering Cancer Center in New York. The targeted therapy would rapidly kill the cancer cells in more patients than would benefit from ipilimumab alone. That wave of destruction might then scatter cellular debris that could catch the attention of an immune system boosted by ipilimumab, yielding a more durable response. "To me this is the area with the most excitement," Allison says. 